\title{
RNA Detection Based on Graphene Field-Effect Transistor Biosensor
}

\author{
Meng Tian, ${ }^{1,2}$ Shicai Xu (D), ${ }^{1}$ Junye Zhang, ${ }^{1}$ Xiaoxin Wang, ${ }^{1}$ Zhenhua Li, ${ }^{1}$ \\ Huilan Liu, ${ }^{1}$ Ruihong Song, ${ }^{1,2}$ Ziheng Yu, ${ }^{1}$ and Jihua Wang ${ }^{1}{ }^{1}$ \\ ${ }^{1}$ Shandong Provincial Key Laboratory of Biophysics, College of Physics and Electronic Information, \\ Dezhou University, Dezhou 253023, China \\ ${ }^{2}$ School of Life Science, Shandong Normal University, Jinan 250014, China \\ Correspondence should be addressed to Shicai Xu; shicaixu@dzu.edu.cn and Jihua Wang; jhw25336@126.com
}

Received 8 December 2017; Revised 17 March 2018; Accepted 24 April 2018; Published 3 June 2018

Academic Editor: Jan A. Jung

Copyright (C) 2018 Meng Tian et al. This is an open access article distributed under the Creative Commons Attribution License, which permits unrestricted use, distribution, and reproduction in any medium, provided the original work is properly cited.

Graphene has attracted much attention in biosensing applications due to its unique properties. In this paper, the monolayer graphene was grown by chemical vapor deposition (CVD) method. Using the graphene as the electric channel, we have fabricated a graphene field-effect transistor (G-FET) biosensor that can be used for label-free detection of RNA. Compared with conventional method, the G-FET RNA biosensor can be run in low cost, be time-saving, and be miniaturized for RNA measurement. The sensors show high performance and achieve the RNA detection sensitivity as low as $0.1 \mathrm{fM}$, which is two orders of magnitude lower than the previously reports. Moreover, the G-FET biosensor can readily distinguish target RNA from noncomplementary RNA, showing high selectivity for RNA detection. The developed G-FET RNA biosensor with high sensitivity, fast analysis speed, and simple operation may provide a new feasible direction for RNA research and biosensing.

\section{Introduction}

The detection of RNA can provide very useful information for molecular biology research [1], genetic disease diagnosis [2], and environmental monitoring [3], which is of great significance in life science. To date, many methods for RNA detection have been developed, such as northern blotting [4], gel electrophoresis [5], and real-time quantitative polymerase chain reaction (QRT-PCR) [6]. However, these methods suffer from many limitations, such as low throughput analysis, expensive reagent, complex operation, time consuming, and poor sensitivity. Therefore, it is necessary to develop a highly efficient, sensitive, fast, and low-cost platform for RNA detection.

In recent years, the significance of biological sensors using nanomaterials, such as silicon nanowires $[7,8]$, carbon nanotubes $[9,10]$, and graphene $[11,12]$, has drawn significant attention to scientific research owing to its unique physical and chemical properties, such as surface effect and microsize effect. It is expected that these biosensors can be used as nextgeneration diagnostic chips for the future, which will play an increasingly important role in life science research and medical testing. Among the nanomaterials, graphene is a twodimensional planar film and has many unique properties, such as high carrier mobility and ease of functionalization $[13,14]$. These unique properties of graphene have unique electrical advantages in building field-effect transistor biosensors for detecting biomolecules.

In this paper, we developed a graphene field-effect transistor (G-FET) biosensor using graphene as an electric channel. The single strand DNA as probe was immobilized on the surface of graphene by using 1-pyrenebutanoic acid succinimidyl ester (PBASE) as a linker. The target RNA is introduced onto the biosensor sample cell to hybridize with probe DNA. The sensor shows high sensitivity for RNA detection. Significant sensor response was achieved for detecting trace amounts of RNA in range from $0.1 \mathrm{fM}$ to $1 \mathrm{pM}$. Moreover, the sensing strategy also shows a high selectivity by performing the contrast experiment. Compared with the traditional RNA detection technology, the G-FET RNA biosensor not only provides fast analysis, simple operation and low cost, but also shows 
high sensitivity and selectivity. This kind of biosensor may provide a new way for RNA biosensing applications.

\section{Materials and Methods}

2.1. Materials. Glass substrate $(30 \times 30 \times 1.1 \mathrm{~mm})$ with indium tin oxides (ITO) electroconductive film was purchased from Hua Nan Xiang Cheng (Shenzhen, China) Ltd. Phosphate buffered saline (PBS) (E607008-0500, pH = 7.2-7.4) was purchased from Sangon Biotech (Shanghai, China) Co., Ltd. $\mathrm{Ag} / \mathrm{AgCl}$ electrode with analytical measuring electrode was purchased from Yancheng Arduino Analytical Instruments (Jiangsu Province, China) Co., Ltd. The HPLC-purified DNA probes and RNAs are synthesized by Sangon Biotech (Shanghai, China) Co., Ltd. RNaseZap was purchased from Sigma-Aldrich. Both DNA probes and RNAs were composed of $15 \mathrm{bp}$, which are dissolved and diluted by $0.1 \mathrm{x}$ PBS buffer. The sequence of single strand probe DNA, fullcomplementary RNA (FC-RNA), and noncomplementary RNA (NC-RNA) was 5'-TGTACATCACAACTA-3', 5'UAGUUGUGAUGUACA- $3^{\prime}$, and $5^{\prime}$-UGCAGCUUAGCUGUA-3', respectively.

2.2. Preparation of Graphene. The graphene films were grown by chemical vapor deposition (CVD) on a copper foil substrate at $1050^{\circ} \mathrm{C}$. Prior to growth, the copper foil was cleaned successively with acetone, ethanol, and deionized water for $20 \mathrm{~min}$ in a KQ5200 ultrasonic cleaner. Then, the copper foil was annealed at $1050^{\circ} \mathrm{C}$ for $10 \mathrm{~min}$ with flowing $\mathrm{H}_{2}$ of $15 \mathrm{sccm}$ at 90 mtorr for improving the quality of graphene $[15,16]$. For graphene growth, the gas mixture of $\mathrm{CH}_{4}$ and $\mathrm{H}_{2}$ is then flowed at 460 mtorr with rates of 16 and $30 \mathrm{sccm}$ for $30 \mathrm{~min}$. Finally, the sample was rapidly cooled to room temperature with flowing $\mathrm{H}_{2}$ of $15 \mathrm{sccm}$ at 90 mtorr.

2.3. Fabrication of G-FETs. In this paper, poly(methyl methacrylate) (PMMA) was used as the intermediate carrier to transfer graphene films $[17,18]$. The PMMA acetone solution was coated on graphene/copper foils with low-speed suspension $9 \mathrm{~s}(1600 \mathrm{r} / \mathrm{min})$ and high-speed spin-coating $60 \mathrm{~s}(3000 \mathrm{r} / \mathrm{min})$, followed by baking at $150^{\circ} \mathrm{C}$ for $30 \mathrm{~min}$. The copper was etched with $\mathrm{FeCl}_{3}$ solution over a period of $10 \mathrm{~h}$ to release PMMA/graphene films into the solution (Figure 1(a)), after washing the PMMA/graphene film with deionized water, which was transferred onto a glass substrate with two ITO electrodes (Figures 1(b)-1(e)). Here, the ITO electrode was used as source and drain electrode, respectively. Then, the substrate/PMMA/graphene was baked on a hot plate at $180^{\circ} \mathrm{C}$ for 30 minutes to remove the moisture and to make the graphene more flat. After that, the substrate/PMMA/graphene was soaked in acetone solution for 24 hours to remove PMMA to leave graphene on the substrate cross source and drain electrode as the electric channel. A sample cell $(\varphi=0.5 \mathrm{~mm})$ was mounted on graphene for RNA solution addition (Figure 1(f)). An Ag/AgCl electrode as reference electrode was inserted into the sample cell to provide gate voltage $\left(V_{\mathrm{gs}}\right)$.

2.4. Immobilization of DNA Probes on Graphene. In order to immobilize the DNA probes on graphene, PBASE is used as an intermediate linker to modify graphene due to its high chemical stability and being nonhydrophilic and insoluble in organic solvents. The pyrene group of PBASE bonds to the six-membered ring structure on the surface of graphene by the $\pi-\pi$ stacking force, and the succinimide can covalently bond with the amino group on the probe DNA. PBASE solution at $1 \mathrm{mM}$ in N,N-dimethylformamide (DMF) was added to the sample cell at room temperature for $1 \mathrm{~h}$ to make PBASE attach on graphene. Then, the G-FET device was washed with DMF and 0.1x PBS buffer sequentially. After that, $100 \mathrm{nM}$ DNA was introduced to the sample cell at room temperature for $18 \mathrm{~h}$ to serve as probe for RNA detection. Finally, the excess unreacted DNA was rinsed with a $0.1 \mathrm{x}$ PBS buffer.

2.5. Detection of RNAs. In order to reduce the degradation of RNAs, all the experiments involved in RNA were carried out in RNase-free environment. All buffers and deionized water were disposed with $0.1 \%$ DEPC overnight and autoclaved. GFET device was treated with RNase Zap reagent before adding RNAs. The target RNAs with different concentrations were added to the sample cell to hybridize with probe DNA at room temperature for $5 \mathrm{~h}$. The target RNAs were rinsed with $0.1 \mathrm{x}$ PBS buffer to avoid nonspecific binding. The transfer curve of G-FET was measured in a semiconductor parameter analyzer (PDA FS360) coupled with a probe station (PEH-4). In the measurement, the G-FET device was provided with a varying gate voltage $V_{\mathrm{gs}}$ from -0.6 to $0.6 \mathrm{~V}$ with the scanning step of $0.025 \mathrm{~V}$ by an $\mathrm{Ag} / \mathrm{AgCl}$ electrode and a constant voltage of $0.01 \mathrm{~V}$ was provided between drain and source electrodes.

2.6. Characterizations. The transferred graphene on glass and the functionalization step were characterized by Raman spectra. The Raman spectra of the graphene were performed using a Raman spectrometer (Horiba HR-800) with laser excitation at $532 \mathrm{~nm}(2.33 \mathrm{eV})$. The excitation laser spot is about $0.5 \mu \mathrm{m}$. A field emission-scanning electron microscope (FE-SEM) (ZEISS, SUPRATM-55) with accelerating voltage $5 \mathrm{kV}$ was used to analyze the morphology of the as-grown graphene on $\mathrm{Cu}$ foil and the transferred graphene on glass.

\section{Results and Discussion}

Figure 2(a) shows SEM image of as-grown graphene film on copper foil. There are a few light ridges observed on the surface of the sample. The ridges were caused by the difference of thermal expansion coefficient between $\mathrm{Cu}$ and graphene [19]. After the graphene was transferred onto the glass substrate, no ridges were observed as shown in Figure 2(b). It could be that the graphene film is spread out in the transfer process. The films are found to be flat and continuous without any holes and tears, indicating the well transfer of graphene. Figure 2(c) shows the Raman spectrum of graphene films after transferred on glass substrate. The D, G, and 2D bands associated with graphene are clearly seen at $\sim 1348 \mathrm{~cm}^{-1}$, $1585 \mathrm{~cm}^{-1}$, and $\sim 2750 \mathrm{~cm}^{-1}$, respectively. Among them, the G band is caused by the first-order scattering of the in-plane optical phonon $E_{2 \mathrm{~g}}$ mode, and the $2 \mathrm{D}$ band is the result of a second-order process involving two phonons with opposite 


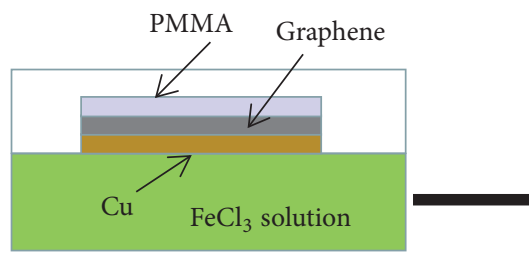

(a)

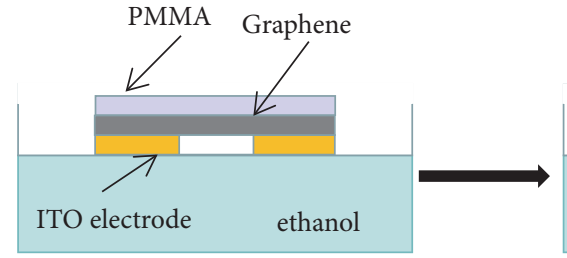

(d)

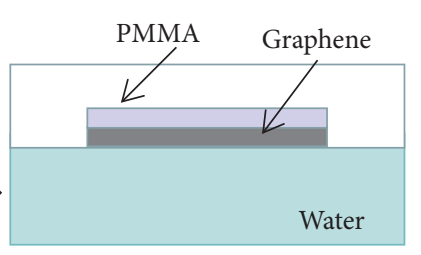

(b)

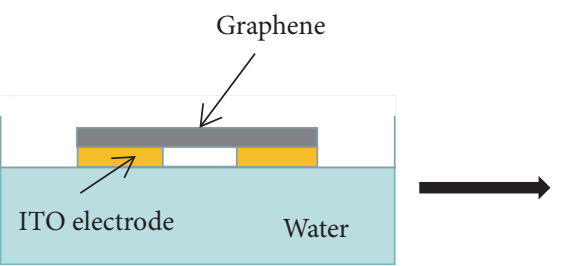

(e)

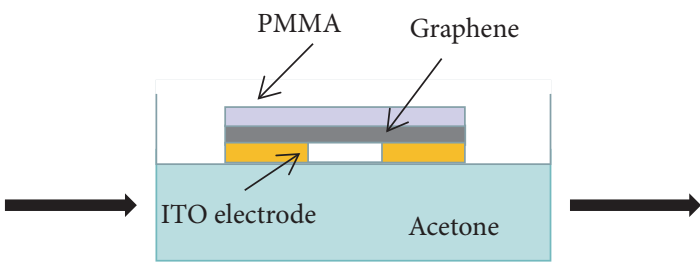

(c)

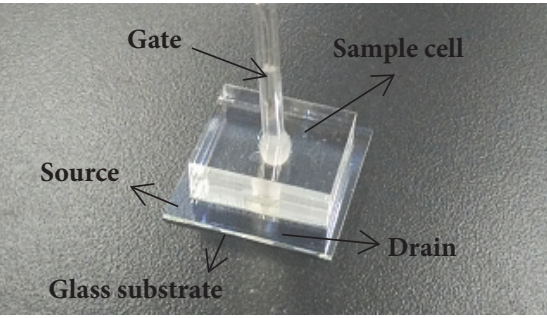

(f)

FIGURE 1: Schematic of the graphene transfer and G-FET fabrication. (a)-(e) Graphene film transferred onto the glass substrate with two ITO electrodes. (f) Photo of the fabricated G-FET.

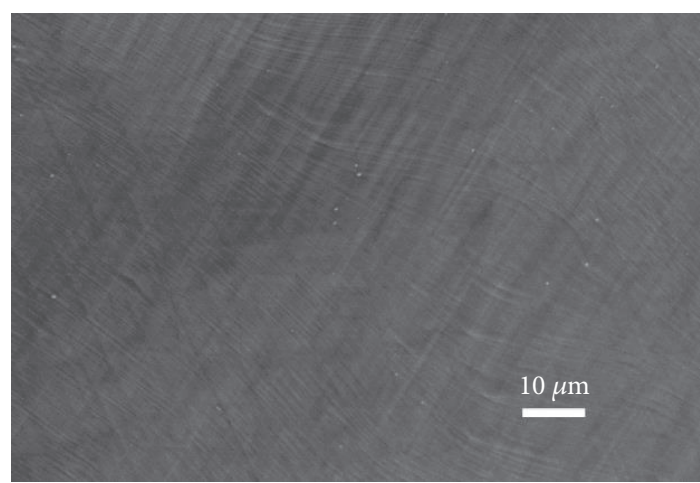

(a)

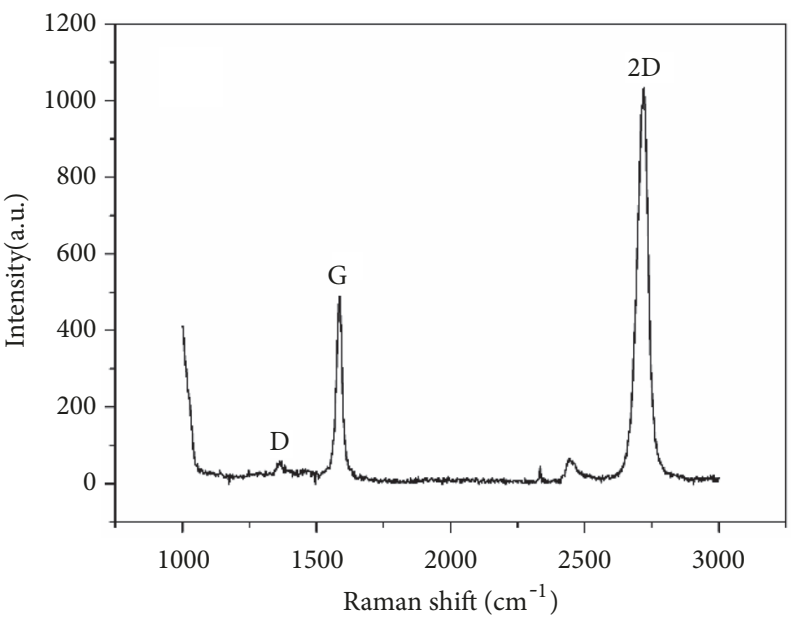

(c)

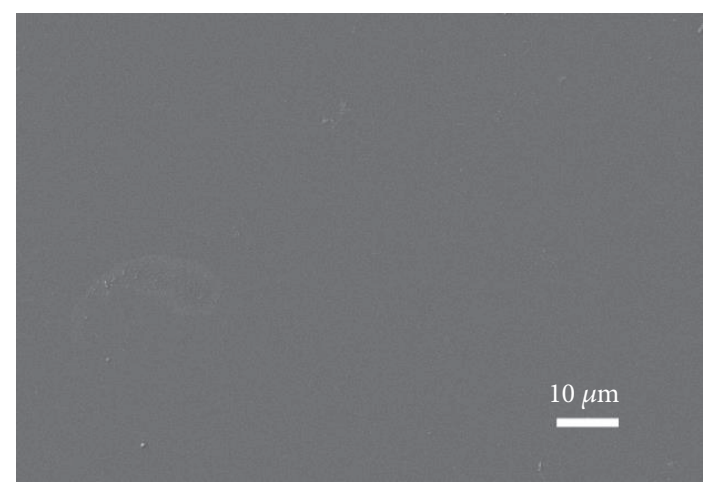

(b)

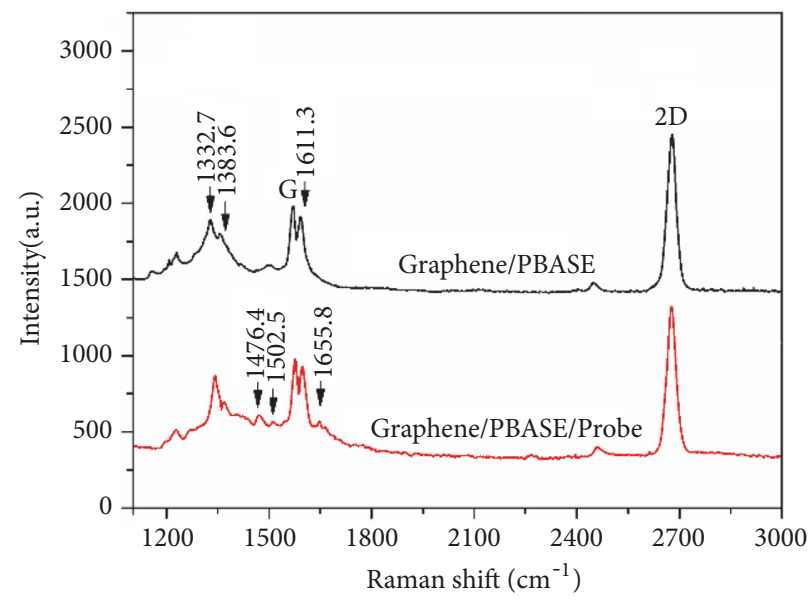

(d)

FIGURE 2: (a) SEM image of the as-grown graphene on the copper foil. (b) SEM image of graphene on glass substrate. (c) Raman spectrum of the graphene on glass substrate. (d) Raman spectra of graphene in the process of functionalization. 


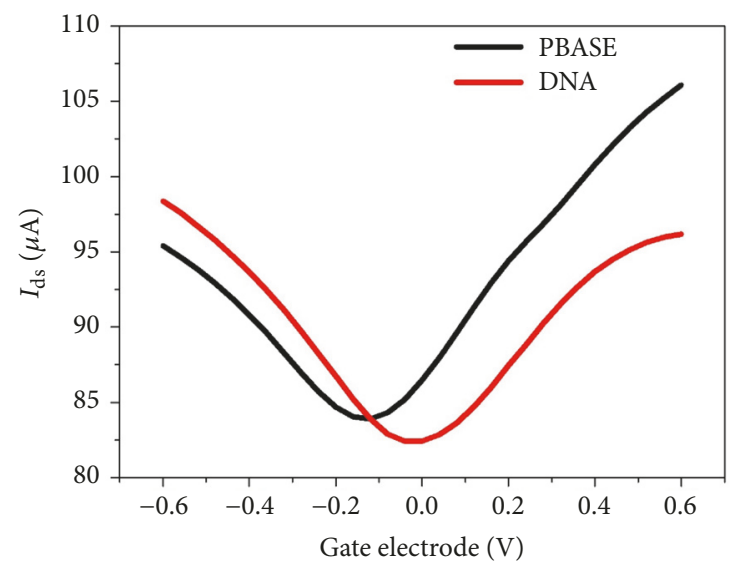

FIgURE 3: The transfer characteristics of the PBASE modified G-FET (black curve) and after probe DNA functionalization (red curve).

momentum, which are regarded as characteristic bands of the graphene structure [20]. The ratio of the intensity $2 \mathrm{D}$ band and $\mathrm{G}$ band $\left(I_{2 \mathrm{D}} / I_{\mathrm{G}}\right)$ is about 2-3, indicating the grown graphene is monolayer. The $\mathrm{D}$ band is due to the out-ofplane breathing mode of the $\mathrm{sp}^{2}$ atoms and is related to the structural defects [21]. Raman spectra record the functionalization step of graphene as shown in Figure 2(d). After introduction PBASE molecules into graphene, several new bands were observed. The bands at 1332.7 and $1383.6 \mathrm{~cm}^{-1}$ can be assigned to the introduction of disorder arising from orbital hybridization of the molecule with the graphene plane, and the bands at $1611.3 \mathrm{~cm}^{-1}$ are due to the pyrene group resonance [22]. After the probe DNA was further immobilized on the graphene/PBASE layer, more bands appeared at $1476.4,1502.5$, and $1655.8 \mathrm{~cm}^{-1}$ which can be assigned to the vibrational modes of DNA molecules [23].

Figure 3 shows the transfer characteristics (drain-source current $\left(I_{\mathrm{ds}}\right)$ versus gate voltage $\left.\left(V_{\mathrm{gs}}\right)\right)$ of the PBASE modified G-FET (black curve) and after probe DNA functionalization (red curve). Here, the drain-source voltage was set to be a small value of $0.01 \mathrm{~V}$ to reduce the electrical damage to probe DNA. After the probe DNA was modified on the GFET by PBASE, the charge neutrality point voltages $\left(V_{\mathrm{cnp}}\right)$ were shifted to the positive gate voltage direction. The change of electric potential of graphene can be explained by the negative electrostatic gating effect. As DNA has a negatively charged triphosphate group, it can modulate the Fermi level of graphene by inducing excess hole carriers and in turn shift $V_{\text {cnp }}$ to the positive direction $[24,25]$.

Figure 4(a) shows the transfer characteristics of DNA probe-modified G-FETs before and after addition of target RNAs (full-complementary) with different concentration from $0.1 \mathrm{fM}$ to $1 \mathrm{pM}$. With the concentration increase of the target RNAs, $V_{\text {cnp }}$ successively shifted to the negative direction. The shift can be explained as an electron transfer effect caused by nonelectrostatic stacking interaction between RNAs and graphene layer [26]. Figure 4(b) shows the $V_{\text {cnp }}$ shift as a function of the concentration of target RNAs. The GFET sensor shows a very high sensitivity. The electrical potential of graphene potential was significantly decreased when the RNA concentration was increased from $0.1 \mathrm{fM}$ to $1 \mathrm{pM}$. It is noted that the detection limit of our G-FET device for RNA is as low as $0.1 \mathrm{fM}$. The detection limit is two orders of magnitude lower than that reported in the literature where the reduced graphene oxide is used as electric channel [14]. Here, the high sensitivity of our G-FET device can be attributed to the excellent transfer properties of CVD graphene. To represent the capability of the quantitative detection of RNA and its reproducibility, the linear fit calibration curve $\left(R^{2}=0.970\right)$ with error bars was also shown in Figure 4(b). The reasonable linear response of G-FET is observed from $0.1 \mathrm{fM}$ to $1 \mathrm{pM}$. However, as indicated by error bars, the fluctuation of sensor response was relatively large, especially for lower concentration detection.

Besides sensitivity, selectivity is also a critical parameter to assess the performance of a sensor. In order to verify the selectivity of the G-FET device for RNA detection, identical concentrations of noncomplementary RNA (NC-RNA) and full-complementary RNA (FC-RNA) were introduced into the sample cell, respectively. As shown in Figure 5, a significant shift of $V_{\text {cnp }}$ occurs when detecting FC-RNA. In contrast, for NC-RNA detection, the shift of $V_{\text {cnp }}$ is negligible. This contrast indicates that only a small amount of NC-RNA binds to the probe DNA by nonspecific adsorption. Therefore, the G-FET biosensor makes a good distinction between target RNA and noncomplementary RNA, indicating the high selectivity of our G-FET RNA sensor.

\section{Conclusions}

In this work, we developed a G-FET biosensor for detecting trace amounts of RNA. The addition of target RNA affects the electrical potential of graphene and allows label-free detection of RNA by readout of the shift of $V_{\text {cnp }}$. Benefiting from the excellent transfer properties of the CVD graphene, the sensing sensitivity of the sensor was significantly enhanced. The detection limit of G-FET biosensor for sensing RNA is as low as $0.1 \mathrm{fM}$, which is two orders of magnitude lower than the previously reports. Moreover, the G-FET biosensor can readily distinguish target RNA from NC-RNA, showing high selectivity for RNA detection. The developed G-FET 


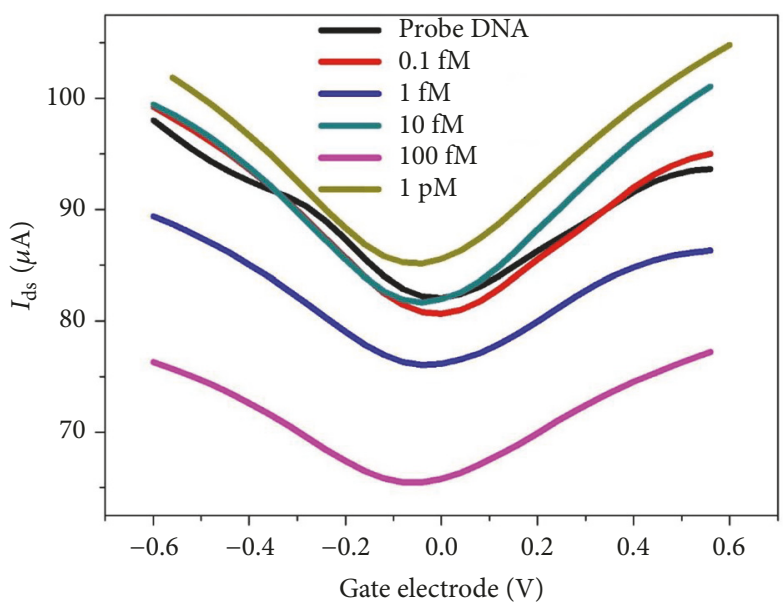

(a)

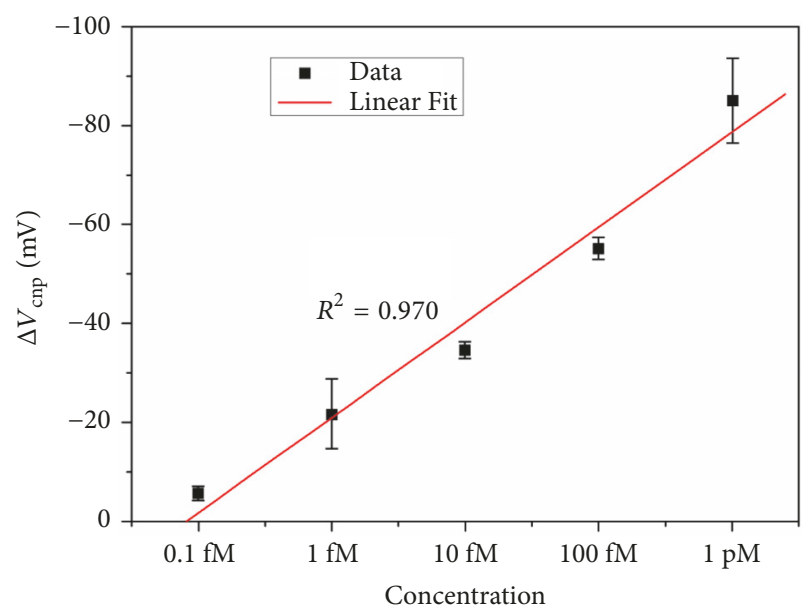

(b)

FIgURE 4: (a) Transfer characteristics of G-FET with addition of different concentrations of target RNA from $0.1 \mathrm{fM}$ to $1 \mathrm{pM}$. (b) The change of $\Delta V_{\text {cnp }}$ of the G-FET with addition of the target RNA from $0.1 \mathrm{fM}$ to $1 \mathrm{pM}$.

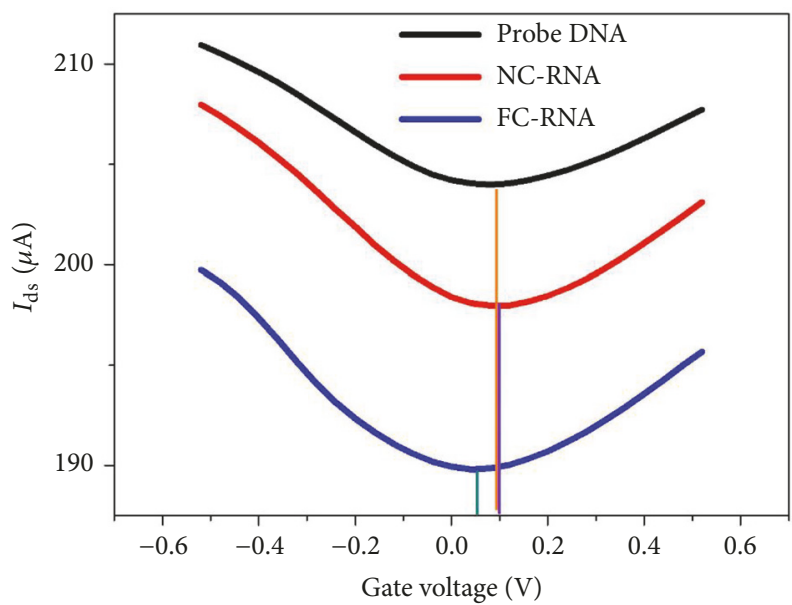

FIGURE 5: The transfer characteristics of the pristine DNA-modified G-FET biosensor (black) and after addition of NC-RNA (red) and FC-RNA (blue).

provides a possible way for label-free and ultrasensitive RNA detection, suggesting a promising future for biosensing applications.

\section{Conflicts of Interest}

There are no conflicts of interest related to this paper.

\section{Acknowledgments}

The authors are grateful for financial support from National Natural Science Foundation of China (11604040, 61671107, and 11704059), Research Foundation for Advanced Talents of Dezhou University (320061), Project of Shandong
Province Higher Educational Science and Technology Program (J15LJ05), Shandong Province Natural Science Foundation (ZR2016FQ08), and Taishan Scholars Program of Shandong Province (Tshw201502045).

\section{References}

[1] D. Janasek, J. Franzke, and A. Manz, "Scaling and the design of miniaturized chemical-analysis systems," Nature, vol. 442, no. 7101, pp. 374-380, 2006.

[2] D. C. Hay Burgess, J. Wasserman, and C. A. Dahl, "Global health diagnostics.", Nature, vol. 444, pp. 1-2, 2006.

[3] J. Mcauley et al., "An assessment of RNA environmental room control monitoring for NAT testing," Transfusion, vol. 41, pp. 29S-29S, 2001.

[4] K. A. Kuznicki, P. A. Smith, W. M. A. Leung-Chiu, A. O. Estevez, H. C. Scott, and K. L. Bennett, "Combinatorial RNA interference indicates GLH-4 can compensate for GLH-1; These two P granule components are critical for fertility in C. elegans," Development, vol. 127, no. 13, pp. 2907-2916, 2000.

[5] A. Petrov, A. Tsa, and J. D. Puglisi, "Analysis of RNA by analytical polyacrylamide gel electrophoresis," Methods in Enzymology, vol. 530, pp. 301-313, 2013.

[6] E. Sueoka, N. Sueoka, K. Iwanaga et al., "Detection of plasma hnRNP B1 mRNA, a new cancer biomarker, in lung cancer patients by quantitative real-time polymerase chain reaction," Lung Cancer, vol. 48, no. 1, pp. 77-83, 2005.

[7] S. Zafar, M. Khater, V. Jain, and T. Ning, "A comparison between bipolar transistor and nanowire field effect transistor biosensors," Applied Physics Letters, vol. 106, no. 6, Article ID 063701, 2015.

[8] M.-Y. Shen, B.-R. Li, and Y.-K. Li, "Silicon nanowire field-effecttransistor based biosensors: From sensitive to ultra-sensitive," Biosensors and Bioelectronics, vol. 60, pp. 101-111, 2014.

[9] K. Maehashi, T. Katsura, K. Kerman, Y. Takamura, K. Matsumoto, and E. Tamiya, "Label-free protein biosensor based on 
aptamer-modified carbon nanotube field-effect transistors," Analytical Chemistry, vol. 79, no. 2, pp. 782-787, 2007.

[10] S. Okuda, S. Okamoto, Y. Ohno, K. Maehashi, K. Inoue, and K. Matsumoto, "Horizontally aligned carbon nanotubes on a quartz substrate for chemical and biological sensing," The Journal of Physical Chemistry C, vol. 116, no. 36, pp. 1949019495, 2012.

[11] Y. Ohno, K. Maehashi, Y. Yamashiro, and K. Matsumoto, "Electrolyte-gated graphene field-effect transistors for detecting $\mathrm{pH}$ and protein adsorption," Nano Letters, vol. 9, no. 9, pp. 33183322, 2009.

[12] K. Maehashi, Y. Sofue, S. Okamoto, Y. Ohno, K. Inoue, and K. Matsumoto, "Selective ion sensors based on ionophore-modified graphene field-effect transistors," Sensors and Actuators B: Chemical, vol. 187, pp. 45-49, 2013.

[13] A. K. Geim and K. S. Novoselov, “The rise of graphene," Nature Materials, vol. 6, no. 3, pp. 183-191, 2007.

[14] B. Cai, L. Huang, H. Zhang, Z. Sun, Z. Zhang, and G.-J. Zhang, "Gold nanoparticles-decorated graphene field-effect transistor biosensor for femtomolar MicroRNA detection," Biosensors and Bioelectronics, vol. 74, pp. 329-334, 2015.

[15] S. C. Xu, B. Y. Man, S. Z. Jiang et al., "Flexible and transparent graphene-based loudspeakers," Applied Physics Letters, vol. 102, no. 15, Article ID 151902, 2013.

[16] M. A. Fanton, J. A. Robinson, C. Puls et al., "Characterization of graphene films and transistors grown on sapphire by metalfree chemical vapor deposition," ACS Nano, vol. 5, no. 10, pp. 8062-8069, 2011.

[17] X. You and J. J. Pak, "Lithography-free fabrication of bistability graphene FET biosensor," in Proceedings of the 2011 11th IEEE International Conference on Nanotechnology, NANO 2011, pp. 617-620, Portland, OR, USA, August 2011.

[18] W. Yue, S. Jiang, S. Xu, and C. Bai, "Fabrication of integrated field-effect transistors and detecting system based on CVD grown graphene," Sensors and Actuators B: Chemical, vol. 195, pp. 467-472, 2014.

[19] X. Li, W. Cai, J. An et al., "Large-area synthesis of high-quality and uniform graphene films on copper foils," Science, vol. 324, no. 5932, pp. 1312-1314, 2009.

[20] V. T. Nguyen, H. Doan Le, V. Chuc Nguyen et al., "Synthesis of multi-layer graphene films on copper tape by atmospheric pressure chemical vapor deposition method," Advances in Natural Sciences Nanoscience Nanotechnology, vol. 4, Article ID 035012, 2013.

[21] A. N. Obraztsov, E. A. Obraztsova, A. V. Tyurnina, and A. A. Zolotukhin, "Chemical vapor deposition of thin graphite films of nanometer thickness," Carbon, vol. 45, no. 10, pp. 2017-2021, 2007.

[22] Y. Zhu, Y. Hao, E. A. Adogla et al., "A graphene-based affinity nanosensor for detection of low-charge and low-molecularweight molecules," Nanoscale, vol. 8, no. 11, pp. 5815-5819, 2016.

[23] E. Papadopoulou and S. E. J. Bell, "Label-free detection of single-base mismatches in DNA by surface-enhanced raman spectroscopy," Angewandte Chemie International Edition, vol. 50, no. 39, pp. 9058-9061, 2011.

[24] S. Xu, J. Zhan, B. Man et al., "Real-time reliable determination of binding kinetics of DNA hybridization using a multi-channel graphene biosensor," Nature Communications, vol. 8, Article ID 14902, 2017.
[25] Y. Ohno, S. Okamoto, K. Maehashi, and K. Matsumoto, "Direct electrical detection of DNA hybridization based on electrolytegated graphene field-effect transistor," Japanese Journal of Applied Physics, vol. 52, no. 11, Article ID 110107, 2013.

[26] Z. Yin, Q. He, X. Huang et al., "Real-time DNA detection using Pt nanoparticle-decorated reduced graphene oxide field-effect transistors," Nanoscale, vol. 4, no. 1, pp. 293-297, 2012. 

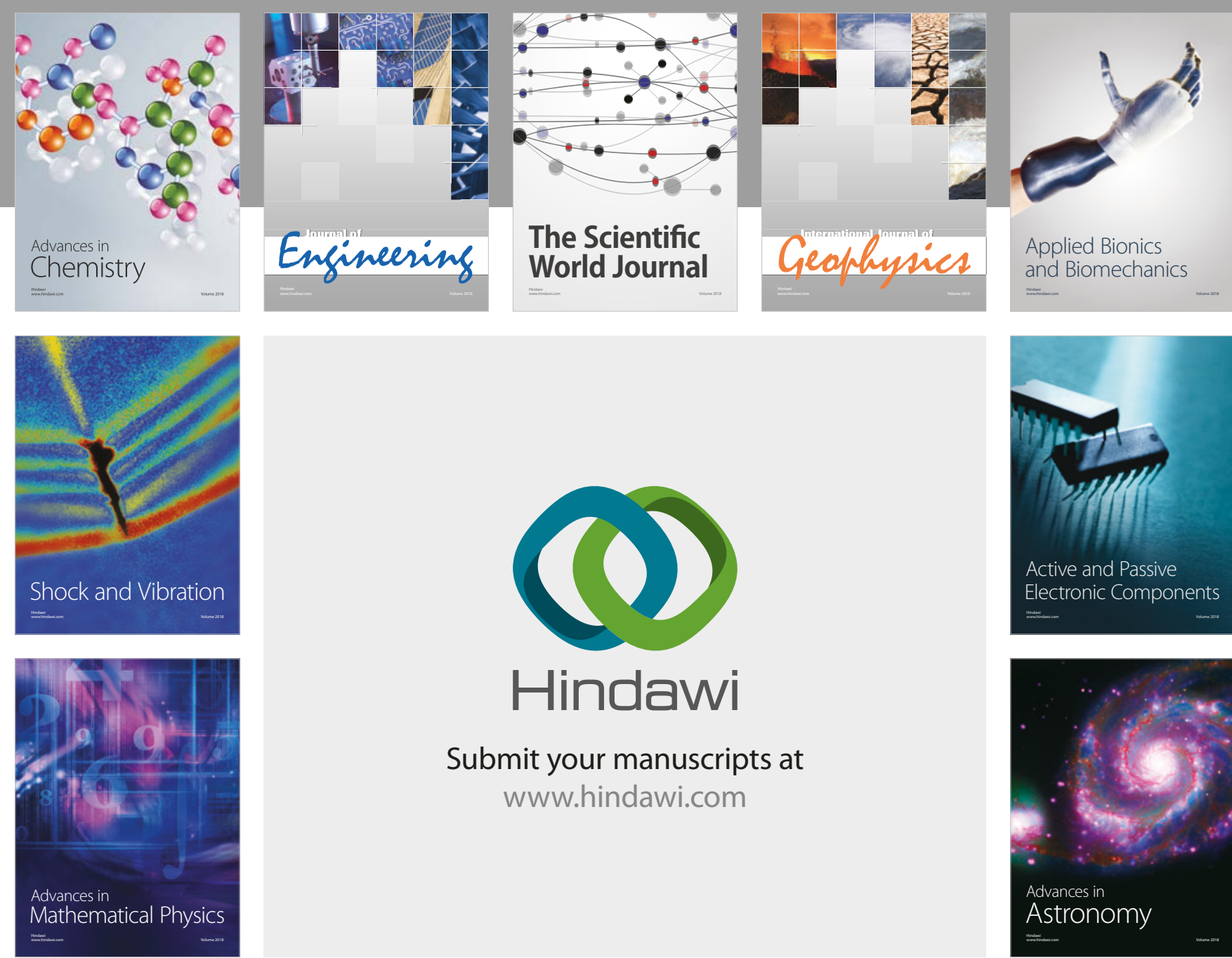

Submit your manuscripts at

www.hindawi.com

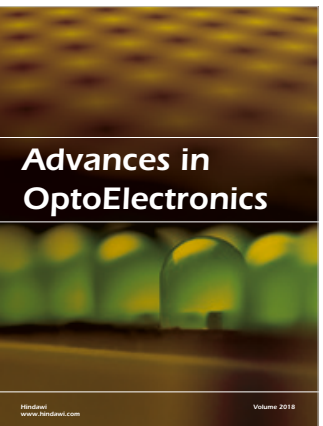

\section{Rotcting Machinery}
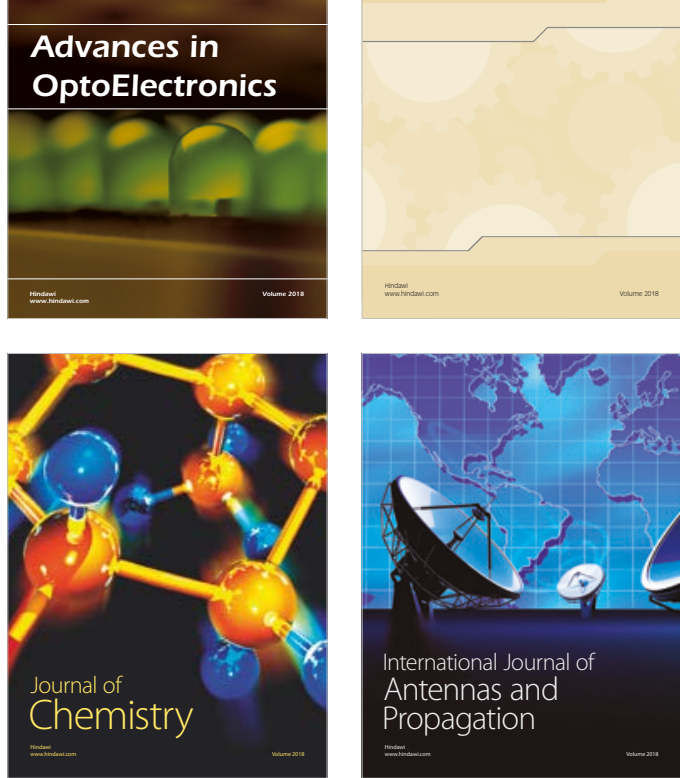

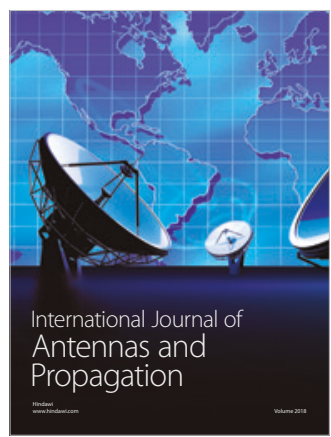

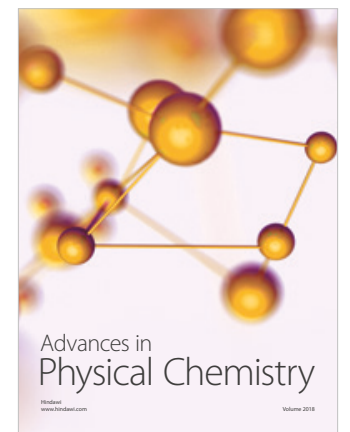

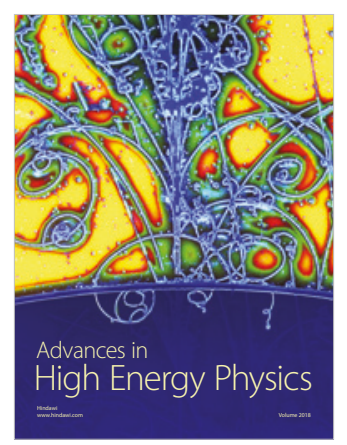

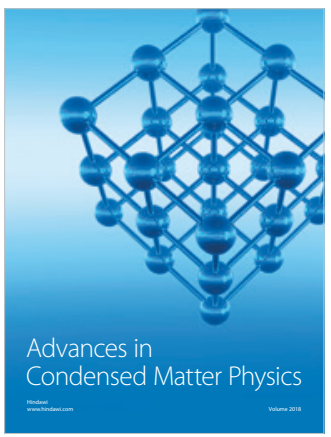

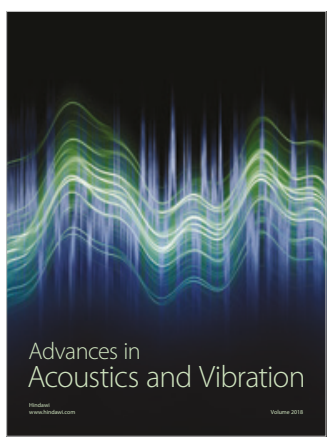

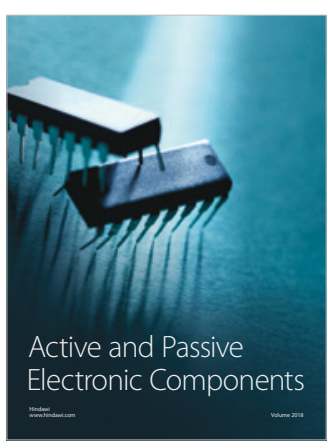
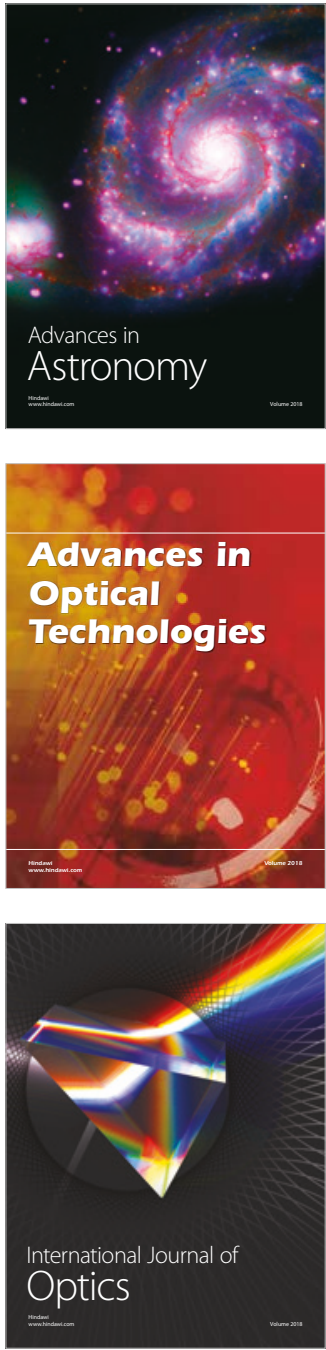